\title{
Study of correlation between serum vit-B12 and homocysteine levels with ischemic heart disease
}

\author{
Dnyaneshwar Malharrao Ghuge ${ }^{1 *}$, Balasaheb Eknathrao Karad ${ }^{2}$
}

${ }^{1}$ Associate Professor, ${ }^{2}$ Professor, Department of Medicine, Pacific Institute of Medical Sciences, Udaipur, Rajasthan, INDIA. Email: drdmghuge@yahoo.com, karadbalasaheb@gmail.com

\begin{abstract}
Background: Homocysteine is regarded as a risk factor for coronary artery disease, stroke, dementia and peripheral vascular disease. Present study aims to identify any correlation between serum vit-B12 and homocysteine levels in patients with ischemic heart disease at a tertiary hospital. Material and Methods: Present study was a prospective, observational study. Cases were selected among patients admitted to the Medicine Intensive Care unit, with symptoms of acute myocardial infarction with or without electrocardiographic signs of elevated ST segment. Age and BMI matched controls were selected from patients attending outpatient clinic, who had no history of cardiovascular disease or other chronic diseases such as renal failure. Results: Total 100 cases and 100 controls (age and BMI) matched controls were considered for present study. Risk factors to ischemic heart disease such as hypertension, diabetes melitus, smoking, sedentary lifestyle, family history for coronary disease and family history for hypertension were significantly more in cases as compared to controls and difference was statistically significant. We noted raised serum Homocysteine levels in cases as compared to controls, difference was statistically significant. Also serum vitamin B12 levels were less in cases as compared to controls, difference was statistically significant. Conclusion: We noted raised serum Homocysteine levels in cases as compared to controls, difference was statistically significant. Also serum vitamin B12 levels were less in cases as compared to controls, difference was statistically significant. vitamin B12 deficiency causing hyperhomocysteinemia may be a risk factor for cardiovascular disease and important for prediction of future cardiovascular disease.

Key Words: hyperhomocysteinemia, ischemic heart disease, Vitamin B12 deficiency
\end{abstract}

\section{*Address for Correspondence:}

Dr Dnyaneshwar Malharrao Ghuge, Associate Professor, Department of Medicine, Pacific Institute of Medical Sciences, Udaipur, Rajasthan, INDIA.

Email: karadbalasaheb@gmail.com

Received Date: 02/12/2019 Revised Date: 10/01/2020 Accepted Date: 13/02/2020

DOI: https://doi.org/10.26611/10211627

This work is licensed under a Creative Commons Attribution-NonCommercial 4.0 International License.

\begin{tabular}{|l|l|}
\hline \multicolumn{2}{|c|}{ Access this article online } \\
\hline Quick Response Code: & Website: \\
\hline & www.medpulse.in \\
& \\
\hline
\end{tabular}

\section{INTRODUCTION}

Vitamin B12 is a water-soluble vitamin needed for normal nerve cell activity, DNA replication, and production of the mood-affecting substance SAMe (S-adenosyl-L- methionine). Vitamin B12 acts with folic acid and vitamin B6 to control homocysteine levels. Homocysteine (Hcy) is a sulfur containing amino acid and is formed during metabolism of methionine, an essential amino acid derived from dietary protein Homocysteine is regarded as a risk factor for coronary artery disease, stroke, dementia and peripheral vascular disease. ${ }^{1}$ Elevated homocysteine level may be due to genetic defects and dietary factors including low intake of vitamin B12, B6 and folic acid. Several studies concluded that increased homocysteine increases the risk of cardiovascular disease through several mechanisms: impaired endothelial function, increased oxidative stress, changes in lipid metabolism, induction of thrombosis. $^{2}$ While, some studies claimed that the measurement and interpretation of levels of homocysteine and other analytes in the days following the acute stroke 
event may also be debatable. ${ }^{3,4}$ Raised homocysteine could be an epiphenomenon of the acute stressful event rather than an actual risk factor for stroke. ${ }^{5}$ Previous hallmark clinical studies on the benefits of nutrients supplementation for decreasing Hcy levels and, in consequence, cardiovascular, cerebrovascular or peripheral artery disease showed contradictory results. ${ }^{6,7}$ Present study aims to identify any correlation between serum vit-B12 and homocysteine levels in patients with ischemic heart disease at a tertiary hospital.

\section{MATERIAL AND METHODS}

Present study was A prospective, observational study conducted in Department of General Medicine, Pacific Institute of Medical Sciences, Udaipur, during June 2019 to November 2019 Approval was taken from ethical committee for present study. Cases were selected among patients admitted to the Medicine Intensive Care unit, with symptoms of acute myocardial infarction with or without electrocardiographic signs of elevated ST segment. Age and BMI matched controls were selected from patients attending outpatient clinic, who had no history of cardiovascular disease or other chronic diseases such as renal failure. Patients with chronic kidney disease, hepatic dysfunction, known endocrinal (except diabetes mellitus) or rheumatologic diseases or chronic infections, and patients being treated with vitamins were excluded from the study. All cases were interviewed for smoking and physical activity. Height, weight were measured and body mass index (BMI) was calculated. Data on clinical history of hypertension (HTN) and diabetes mellitus (DM) and medications (antihypertensive, lipid lowering, and oral hypoglycemic agents) were also noted. Informed consent was taken from subjects. Venous blood samples from the cases were obtained at baseline from 8-12 hours after symptom presentation, and in OPD from controls and plasma was immediately obtained by centrifugation at $4{ }^{\circ} \mathrm{C}$, for 15 minutes at $3000 \mathrm{rpm}$. Statistical analysis was carried out using SPSS Version 23 (Chicago, IL, USA). Data are presented as mean $\pm \mathrm{SD}$, median (range) or number (\%) unless specified. All parametric data were analyzed by Student's t test. All non parametric data were analyzed by chi-square test. A p-value of $<0.05$ was considered statistically significant.

\section{RESULTS}

Total 100 cases and 100 controls (age and BMI) matched controls were considered for present study. Risk factors to ischemic heart disease such as hypertension, diabetes melitus, smoking, sedentary life- style, family history for coronary disease and family history for hypertension were significantly more in cases as compared to controls and difference was statistically significant.
Table 1: Baseline characteristics and prevalence of cardiovascular risk factors

\begin{tabular}{|c|c|c|c|}
\hline Variable & Cases & Controls & p value \\
\hline \multicolumn{4}{|l|}{ Age (years) } \\
\hline Total & $54.2 \pm 8.5$ & $54.6 \pm 9.8$ & 0.72 \\
\hline Males & $51.6 \pm 7.3$ & $51.2 \pm 8.2$ & 0.71 \\
\hline Females & $56.2 \pm 7.9$ & $56.6 \pm 8.3$ & 0.76 \\
\hline Body mass index $(\mathrm{Kg} / \mathrm{m} 2)$ & $25.7 \pm 3.9$ & $25.6 \pm 4.6$ & 0.63 \\
\hline \multicolumn{4}{|l|}{ Risk factor } \\
\hline Hypertension (\%) & 67 & 8 & 0.012 \\
\hline Diabetes melitus (\%) & 29 & 5 & 0.021 \\
\hline Smoking & 32 & 9 & 0.032 \\
\hline Sedentary Life- style (\%) & 35 & 19 & 0.023 \\
\hline $\begin{array}{l}\text { family history for } \\
\text { coronary disease (\%) }\end{array}$ & 59 & 36 & 0.036 \\
\hline $\begin{array}{l}\text { family history for } \\
\text { hypertension (\%) }\end{array}$ & 52 & 33 & 0.039 \\
\hline
\end{tabular}

We noted raised serum Homocysteine levels in cases as compared to controls, difference was statistically significant. Also serum vitamin B12 levels were less in cases as compared to controls, difference was statistically significant.

Table 2: Comparison of Homocysteine and vitamin B12 levels

\begin{tabular}{cccc} 
& Cases & Controls & p-value \\
\hline $\begin{array}{c}\text { Homocysteine } \\
\mu \mathrm{mol} / \mathrm{L}\end{array}$ & $18.21 \pm 6.94$ & $9.13 \pm 6.14$ & 0.001 \\
$\begin{array}{c}\text { Vitamin } \\
\text { B12 pg/ml }\end{array}$ & $356.25 \pm 104.27$ & $467.75 \pm 167.57$ & 0.012 \\
\hline
\end{tabular}

\section{DISCUSSION}

Vitamin B12 is mainly present in animal proteins, particularly in organ meat especially in liver and to a lesser extent in sea foods and dairy products. In Indians, low plasma vitamin B12 has been attributed to vegetarianism as found in food consumption surveys. ${ }^{8,9}$ Low vitamin B12 concentration and hyperhomocysteinemia (HHC) are common in Indian men, particularly in vegetarians and urban residents. ${ }^{10}$ A number of studies have found that Indians and South East Asians including those living in USA have higher levels of homocysteine compared to Americans and Europeans, which translates into 2-4 times higher risk of cerebrovascular disease. ${ }^{11,12}$ In elderly individuals there is atrophy of gastric mucosa, reduction of gastric acid and pepsin secretion resulting in reduced B12 absorption. This may result in elevated homocysteine levels in elderly. Chronic alcohol consumption is associated with elevated homocysteine levels, probably because of the effect of alcohol on vitamin levels. In contrast, moderate alcohol consumption appears to be associated with low homocysteine levels. ${ }^{8}$ The evidence for a causal link between HHC and stroke has also been strengthened by a meta-analytic study based on the principle of Mendelian randomization. ${ }^{13}$ Some hospital- or community-based studies from India have also shown a 
high prevalence of B12 deficiency in both rural and urban population. ${ }^{10,14}$ Singh $\mathrm{D}^{15}$ noted that serum Homocysteine concentration was found to be significantly high in cigarette smokers as compared to non-smokers. In present study smoking was significantly more in cases. Cigarette smoking is known to be associated with a raised plasma Homocysteine level. Smokers also tend to have lower levels of B vitamins, folate, vitamin B6 and vitamin B12, all of which affect Homocysteine levels by acting as cofactors (vitamin B6 and B12) or co-substrate (folate) for the enzymes controlling Homocysteine metabolism. ${ }^{16}$ In an Iranian study, there was no significant difference in mean serum vit B12, tHcy and folate between the ischemic and non ischemic groups. Yet after adjusting for confounding factors including age, sex, BMI, smoking and cholesterol using Multiple Logistic Regression model, the relation remained nonsignificant. ${ }^{17}$ Jagota $\mathrm{D}$ et al. noted a significant relationship between hyperhomocysteinemia, Vit B12 deficiency and folate deficiency with ischemic and nonischemic group. There was significant correlation between Hcys, Folic Acid and Vit B12 levels in CHD patients. ${ }^{18}$ Similar findings were noted in present study. Vitamin B12 deficiency and hyperhomocysteinemia were associated with traditional and non-traditional cardiovascular risk factors and were independently associated with dyslipidemia even after adjustment for all other risk factors in Indian patients with CAD. Thus, vitamin B12 deficiency causing hyperhomocysteinemia may be a risk factor for cardiovascular disease and important for prediction of future cardiovascular disease. ${ }^{19}$ However, a systematic review of cohort studies showed limited evidence of vitamin B12 deficiency and morbidity and mortality from cardiovascular disease, and a metaanalysis of several studies done with vitamin B12 and folate supplementation failed to show a decrease in coronary artery events..$^{20,21}$ The possible management is to use vitamin B for patient risk management. and long-term folate acid-based vitamin therapy is independently associated with reduced mortality in hyperhomocysteinemic patients with cardiovascular disease, and with a decrease in homocysteine concentration. ${ }^{22}$ Larger studies are required to confirmed these patients.

\section{CONCLUSION}

We noted raised serum Homocysteine levels in cases as compared to controls, difference was statistically significant. Also serum vitamin B12 levels were less in cases as compared to controls, difference was statistically significant. vitamin B12 deficiency causing hyperhomocysteinemia may be a risk factor for cardiovascular disease and important for prediction of future cardiovascular disease.

\section{REFERENCES}

1. Kamdi SP, Palkar P. Prevalence of hyperhomocysteinemia in healthy Indian doctors. Bioinformation 2013;9:193-6.

2. Nair,K.G., Nair,S.R, Ashavaid, T.F, Dalal, J.J. and Eghlim F.F., Methylenetetrahydrofolate reductase gene mutation and hyperhomocysteinemia as a risk factor for coronary heart disease in the Indian population, J Assoc Physicians India. 2002, 50:9-15.

3. Panigrahi I, Chatterjee T, Biswas A, Behari M, Choudhry $\mathrm{PV}$, Saxena R, et al... Role of MTHFR C677T polymorphism in ischemic stroke. Neurol India 2006;54:48-50.

4. Salemi G, Gueli MC, D'Amelio M, Saia V, Mangiapane $\mathrm{P}$, Aridon $\mathrm{P}$, et al... Blood levels of homocysteine, cysteine, glutathione, folic acid, and Vitamin B12 in the acute phase of atherothrombotic stroke. Neurol Sci 2009;30:361-4.

5. Haapaniemi E, Helenius J, Soinne L, Syrjälä M, Kaste M, Tatlisumak $\mathrm{T}$, et al... Serial measurements of plasma homocysteine levels in early and late phases of ischemic stroke. Eur J Neurol 2007;14:12-7

6. Bonaa K., et al... "Homocysteine Lowering and Cardiovascular Events after Acute Myocardial Infarction". The New England Journal of Medicine 354 (2006): 15781588 .

7. The Heart Outcomes Prevention Evaluation (HOPE) 2 Investigators. "Homocysteine Lowering with Folic Acid and B Vitamins in Vascular Disease". The New England Journal of Medicine 354 (2006): 1567-1577.

8. Refsum $\mathrm{H}$, Yajnik $\mathrm{CS}$, Gadkari $\mathrm{M}$, et al... Hyperhomocysteinemia and elevated methylmalonic acid indicate a high prevalence of cobalamin deficiency in Asian Indians. Am J Clin Nutr 2001;74: 233-41.

9. Puri A, Gupta OK, Dwivedi RN, Bharadwaj RP, Narain VS, Singh S. Homocysteine and lipid levels in young patients with coronary artery disease. J Assoc Physicians India 2003;51: 681-5.

10. Yajnik CS, Deshpande SS, Lubree HG, Naik SS, Bhat DS, Uradey BS, Deshpande JA, Rege SS, Refsum H, Yudkin JS. Vitamin B12 deficiency and hyperhomocysteinemia in rural and urban Indians. J Assoc Physicians India 2006;54:775-82

11. Hughes K, Ong CN. Homocysteine, folate, vitamin B12, and cardiovascular risk in Indians, Malays, and Chinese in Singapore. J Epidemiol Community Health 2000;54:31-4.

12. Balarajan R. Ethnic differences in mortality from ischaemic heart disease and cerebrovascular disease in England and Wales. BMJ 1991;302:560-4.

13. Casas JP, Bautista LE, Smeeth L, Sharma P, Hingorani AD. Homocysteine and stroke: Evidence on a causal link from mendelian randomisation. Lancet 2005;365:224-32.

14. Khanduri U, Sharma A, Joshi A. Occult cobalamin and folate deficiency in Indians. Natl Med J India 2005;18:182-3.

15. Singh D, Effect of Cigarette Smoking on Serum Homocysteine and Vitamin B12 Level in Male Population of Udaipur. Biochem Anal Biochem 2016, 5: 282.

16. Pagán K, Hou J, Goldenberg RL, Cliver SP, Tamura T, Effect of smoking on serum concentrations of total homocysteine and $\mathrm{B}$ vitamins in mid-pregnancy. Clin Chim Acta 2001, 306: 103-109. 
17. Bandarian, F. et al... "Association of Serum Homocysteine and Coronary Heart Disease in an Iranian Urban Population." Acta Cardiologica Sinica 25 (2009): 142-146.

18. Gagandeep Jagota, Rajinderjit Singh Ahi and Rakendra Singh, Association of Serum Homocysteine and Coronary Heart Disease in North Indian Population, International Journal of Biotechnology and Biomedical Sciences, Volume 5, Issue 1; January-June, 2019 pp. 1-3

19. Mahalle N, Kulkarni MV, Garg MK, Naik SS. Vitamin B12 deficiency and hyperhomocysteinemia as correlates of cardiovascular risk factors in Indian subjects with coronary artery disease. J Cardiol. 2013 Apr;61(4):289-94.
20. Rafnsson SB, Saravanan P, Bhopal RS, Yajnik CS. Is a low blood level of vitamin B12 a cardiovascular and diabetes risk factor? A systematic review of cohort studies. Eur J Nutr (Germany) 2011;50:97-106.

21. Huang T, Chen Y, Yang B, Yang J, Wahlqvist ML, Li D. Meta-analysis of B vitamin supplementation on plasma homocysteine, cardiovascular and all-cause mortality. Clin Nutr 2012;31:448-54.

22. Lonn E, Held C, Arnol JM, et al..., 2006, Rationale, design and baseline characteristics of a large, simple, randomized trial of combined folate acid and vitamins B6 and B12 in high-risk patients: the Heart Outcomes Prevention Evaluation (HOPE)-2 trial. Can J Cardiol ;22:47-53.

Source of Support: None Declared

Conflict of Interest: None Declared 